\title{
Identifiability of the heat transfer coefficient in buildings with unheated spaces
}

\author{
Sarah Juricic ${ }^{1}$, Peder Bacher ${ }^{2}$, Jeanne Goffart ${ }^{1}$, Simon Rouchier $^{1}$, Aurélie Foucquier ${ }^{3}$, \\ Gilles Fraisse $^{1}$ \\ ${ }^{1}$ Univ. Savoie Mont-Blanc, CNRS, LOCIE, Chambéry, France \\ ${ }^{2}$ Technical University of Denmark, DTU Compute, Copenhagen, Denmark \\ ${ }^{3}$ Univ Grenoble Alpes, CEA, LITEN, INES, Grenoble, France
}

\begin{abstract}
In-situ non-intrusive data to identify the heat loss coefficient (HTC) of a building is promising but possibly not sufficiently informative, let alone for disaggregating losses through separate parts of the envelope.

This paper presents a numerical procedure to assess the ability of models to accurately identify overall and disaggregated heat transfers. The procedure is applied to linear regression models in the case of buildings with unheated basements.

The results suggest that only the simplest model yields satisfactory HTC estimates, yet not in all cases. None of the tested models could disaggregate heat losses to the basement. Finally, usual statistical tools for model selection are no guarantee for identifiability of the HTC.
\end{abstract}

\section{Introduction}

The generalization of non intrusive monitoring and wireless sensors is an opportunity for the estimation of the actual energy performance of buildings during operation. The advantage of non intrusive measurements is that they do not impede occupancy, and can serve for a diagnosis that may allow optimal retrofitting decisions. There are however several drawbacks to using in-use data to these objectives. The main drawback is that not all operating conditions of the building are usually known: performance estimation cannot rely on controlled conditions. Furthermore, the number of sensors is usually low, for example only indoor air temperature for the heated space, leading to less informative measurements and possible larger uncertainties.

In this frame, characterizing the energy performance of buildings with unheated adjacent spaces, such as an unheated basement, becomes challenging. Indeed, heat losses towards the ground cannot be monitored but have a significant influence on the overall performance of the building.

Exploiting poorly informative data can be done with linear regressions. Their simplicity will most probably avoid overfitting the data. At the same time, these models aggregate complex dynamical physical phenomena into a few steady-state parameters and are known to result, in some cases, in high uncertainties (Janssens, 2016). The existing literature (Stamp et al., 2013; Jack et al., 2018; Farmer et al., 2016) on numerical and field coheating tests, which are based on regression models, has already shown that particular weather conditions combined with higher thermal mass and specific glazing properties may lead to errors over $10 \%$ of the heat loss coefficient (HTC) estimation. A similar linear disaggregation method (Chambers and Oreszczyn, 2019) recently demonstrated robustness of the estimation of an overall heat power loss coefficient to climate variations and occupant presence, but did not tackle the issue of estimating losses to the ground, assuming them negligible. Their hypothesis could not be checked as the collected data would no allow it. However, on the basis of coheating tests analysis, Bauwens and Roels (2017) found that large temperature differences between adjacent neighbouring zones lead to significant errors in the HTC estimation. There is therefore little doubt that losses to an unheated basement would do the same.

The present paper aims at assessing the ability of several regression models to estimate the global HTC in presence of unheated adjacent spaces. The developed methodology is based on the assessment of the practical identifiability of the HTC: any change in the real value of a building envelope property should be followed by a change in value of the ad hoc coefficient. A numerical framework to test the identifiability of a model and its coefficients is demonstrated in the present paper. The framework is applied to three linear regression models using non intrusive data from a virtual building with an unheated basement. The target is the identification of the overall HTC, and if possible, the disaggregation of heat loss towards the basement. 


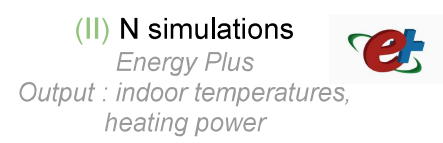

heating power

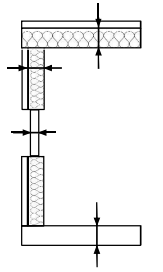

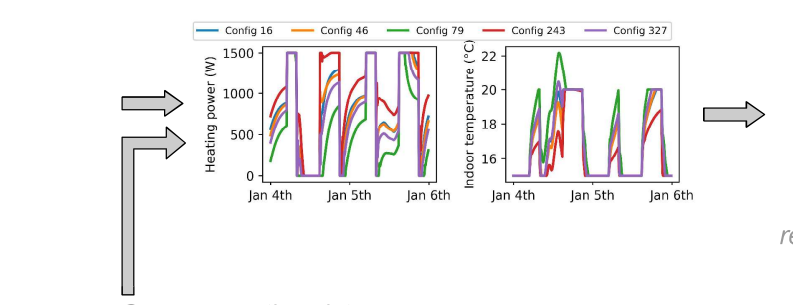
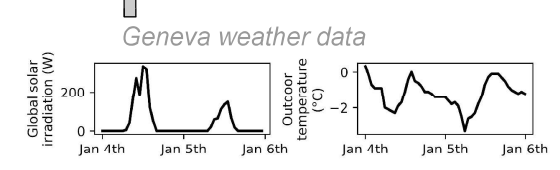

Figure 1: Numerical procedure.
For each regression model (IV) $\mathrm{N}$ estimations of each

(III)

$\mathrm{N}$ least square estimations of several

linear regression models

regression model 1 , regression model 2 ,

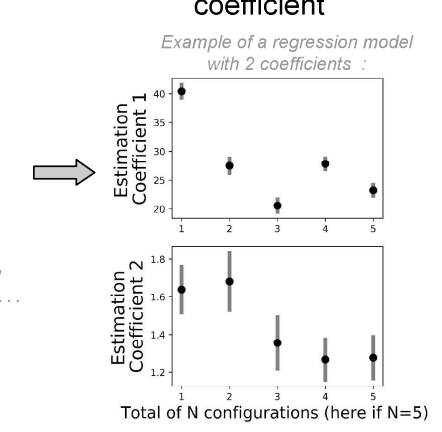

\section{Method}

\section{General numerical framework}

A 4-step method is proposed to assess the identifiability of regression models. It is summarised by Figure 1. Supposing that a given model $\mathcal{M}$ is to be tested:

- (I) First, a computer based model serving as reference model of a single-zone building with an unheated basement was implemented in Energy Plus. Basing the study on a numerical simulation allows changing its thermal properties at will: a total number of $N$ configurations is generated by varying various construction properties (see the description of the case study below).

- (II) A dynamical simulation is run for each of the $N$ configurations. All simulations use the same boundary conditions.

- (III) The reduced-order model $\mathcal{M}$ is trained and its parameters estimated from each of the $N$ outputs of the reference dynamical model.

- (IV) As each parameter of the model $\mathcal{M}$ has been estimated $N$ times, we can compare its variability with the variability of each of the properties of the reference model. This allows assessing whether parameters of the simplified model represent what they should. This paper focuses on the coefficients illustrating heat transfer between the heated space, the unheated basement and the outdoor ambiance.

The novelty of the present procedure lies in the $N$ configurations of the reference model. The existing literature on identifiability assessment based on synthetic data either relies on a single set as in Deconinck and Roels (2017) or at best on a basic design of experiments with a one factor at a time approach as in Senave et al. (2017).

We propose to generate building configurations as to cover as many cases as possible while still being able to assess identifiability and uncover first and second order effects between parameter estimation and building configuration. These are the reasons why the 450 samples are generated by a Latin Hypercube Sampling (LHS) that then allows performing a sensitivity analysis with the RBD-FAST method as detailed below.

\section{Assessing the identifiability of the HTC}

Data based identifiability of the HTC relies on two necessary conditions : practical identifiability of the ad hoc model parameter, i.e. unicity of its estimation as meant in (Maiwald et al., 2011) as well as physical interpretability of the estimation in the sense of (Deconinck and Roels, 2017).

It is a data dependent property: some parameters of a model $\mathcal{M}$ may be identifiable in a test, and non-identifiable in another. The distinction between unicity and interpretability implies that neither unicity of parameter estimates nor finite confidence intervals are a guarantee that their value can be physically interpreted, even if the model is built according to the laws of physics.

The building physics dynamics described in linear regression models are very simplified and the identifiability of their parameters is therefore not guaranteed. Each data-based learned coefficient could indeed aggregate several physical properties and phenomena, in which case the interpretation of their value is not allowed. By using a virtual building as reference, we can assess whether estimated heat transfer coefficients are representative of their reference property.

The general formulation of the theoretical heat loss coefficient follows the usual ISO 13789 standard (ISO, 2017b) and the theoretical losses through the unheated basement follow the ISO 13370 (ISO, 2017a)

$$
H T C=H_{t r}+H_{v}=H_{d}+H_{u}+H_{v}
$$

\section{Case study}

The numerical procedure is based on simulated data from a computer based building model (see Figure 1). This reference model is a single zone building inspired 
Table 1: Heat transfer coefficients according to ISO (2017b).

\begin{tabular}{ll} 
HTC & Global heat transfer coefficient (W/K) \\
$H_{t r}=H_{d}+H_{u}$ & Transmission heat transfer coefficient \\
$H_{v}$ & Ventilation and air infiltration heat transfer coefficient \\
$H_{d}$ & Direct transmission between the heated space and outdoor \\
$H_{u}$ & Transmission through unconditioned adjacent spaces \\
\hline
\end{tabular}

Table 2: Properties of the physical model to be sampled.

\begin{tabular}{lllr}
\hline & Property & Unit & Bounds \\
\hline$R_{g}$ & Ground floor thermal resistance & $\mathrm{W} /\left(\mathrm{m}^{2} . \mathrm{K}\right)$ & {$[1 ; 15]$} \\
$e_{\text {wall }}$ & Wall insulation thickness & $\mathrm{m}$ & {$[0.05 ; 0.20]$} \\
$e_{\text {roof }}$ & Roof insulation thickness & $\mathrm{m}$ & {$[0.05 ; 0.20]$} \\
$h_{w}$ & Window height & $\mathrm{m}$ & {$[0.5 ; 1]$} \\
$\alpha_{g}$ & Exterior ground reflectance & & {$[0.1 ; 0.4]$} \\
$\alpha_{w}$ & Exterior wall reflectance & & {$[0.3 ; 0.7]$} \\
$\tau$ & Solar transmittance of glazing & {$[0.4 ; 0.8]$} \\
\hline
\end{tabular}

by the Bestest case 600 (Judkoff and Neymark, 1995) and shown on Figure 2. It is $8 \mathrm{~m}$ long and $6 \mathrm{~m}$ wide, flat roofed, with one south facing window. It is equipped with a ventilated unheated basement. The heated space itself is not ventilated, as to focus on thermal losses by transmission only. The heated space follows a temperature pattern with a daytime temperature setpoint at $20{ }^{\circ} \mathrm{C}$ and a night time setback at $15{ }^{\circ} \mathrm{C}$.

We study the impact of variations of seven physical properties of the computer based model, listed in Table 2. They concern thermal properties of the envelope, solar gains and heat transfer by radiation. A LHS scheme generates $N=450$ sets of these properties within the bounds shown in Table 2, before running $N$ simulations of the model. The choice of generating 450 samples is driven by the convergence of the sensitivity indices as explained below.

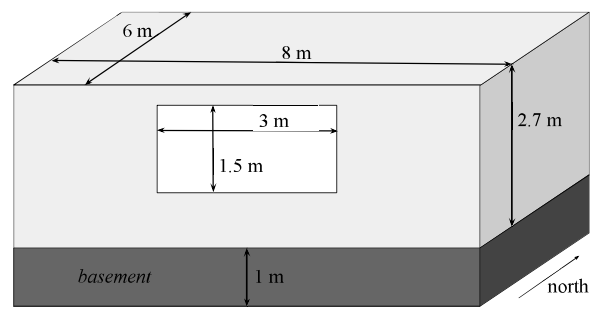

Figure 2: Reference building model.

All 450 configuration are run with Energy Plus from November 1st to February 28th. To be as realistic as possible, the Geneva IWEC weather data file was used for the simulations (ASHRAE, 2001). The regressions are however performed on a smaller data set of 50 days from January $4^{\text {th }}$ to February $12^{\text {th }}$ for the following reasons. First, to prevent the warm-up performed by Energy Plus from affecting this part of the data set. Second, this period is representative of winter conditions with temperature differences between the heated space and the outdoors from $10 \mathrm{~K}$ to $25 \mathrm{~K}$. Furthermore, this period has a majority of cloudy days, which means lower solar gains, more constant indoor temperatures, thus less impact of thermal storage, which is a phenomena not taken into account in the regression models (Bacher and Madsen, 2011).

After simulation by the reference model, the input and output data is averaged daily, from 6 am to 6 am to enable charge and discharge of energy from possible solar gains (Everett, 1985).

\section{Linear regression models}

After $N=450$ configurations have been defined and $N$ virtual datasets have been generated, we study three linear regression models in order to assess their ability to distinguish heat transfer through the unheated basement.

The three models are specified in Table 3 with the following notations: the model output $Q_{h}$ is the heating supply of the conditioned space $(\mathrm{W}) ; T_{i}$, $T_{e}$ and $T_{u}$ are the indoor, outdoor, and unheated space temperatures; $I_{g l o}$ is the global horizontal solar irradiation $\left(\mathrm{W} / \mathrm{m}^{2}\right)$. In addition to the heat transfer coefficients defined in Table 1, model parameters include the solar aperture $A_{\text {sol }}$ and an intercept $c$.

- Model 1 has no coefficient representing the losses through the basement. Its first coefficient aggregates all heat transfers and must be compared to an overall heat transfer coefficient HTC.

- Model 2 has an intercept, supposed to represent constant losses to a neighbouring space with almost constant temperatures, as have basements. Its first coefficient should be compared to $H_{d}$.

- Model 3 explicitly accounts for the temperature of the basement. It therefore includes both coefficients $H_{d}$ and $H_{u}$. The estimation of the HTC follows Equation 2. 
Table 3: Linear regression models under investigation.

Model $1 \quad Q_{h}=\operatorname{HTC}\left(T_{i}-T_{e}\right)+A_{\text {sol }} I_{g l o}$

Model $2 \quad Q_{h}=H_{d}\left(T_{i}-T_{e}\right)+A_{\text {sol }} I_{\text {glo }}+c$

Model $3 \quad Q_{h}=H_{d}\left(T_{i}-T_{e}\right)+H_{u}\left(T_{i}-T_{u}\right)+A_{\text {sol }} I_{g l o}$
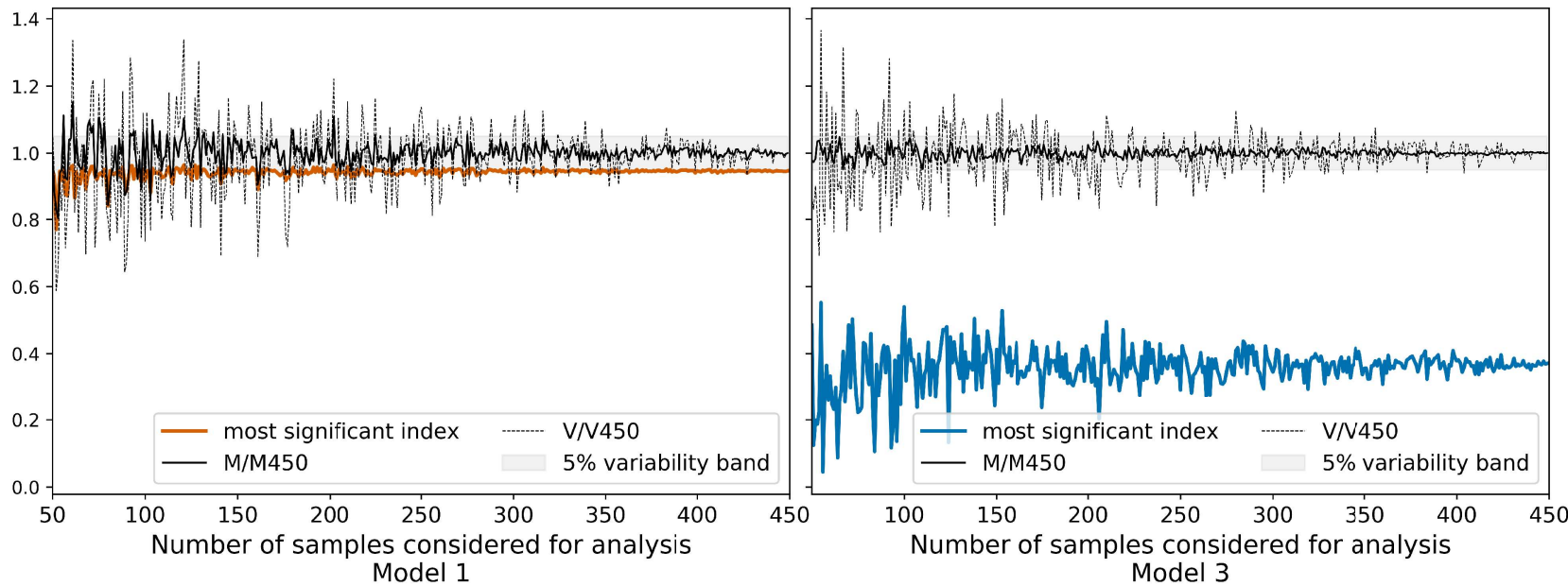

Figure 3: Convergence plot of the means, the variances and the most significant indices on errors of the HTC estimation for Models 1 and 3.

$$
\widehat{H T C}=\widehat{H_{d}}+\widehat{H_{u}} \frac{T_{i}-T_{u}}{T_{i}-T_{e}}
$$

The models will also be compared with the usual criteria used for linear regression:

- The residuals are expected to have white noise properties if a model explains sufficiently the data (Madsen, 2007). If the residuals show unsatisfactory results, the model cannot explain the variability of the data and needs refinement.

- The adjusted $R_{a d j}^{2}$ assesses the model fit as would do a regular $R^{2}$ coefficient, but takes into account the number of independent variables. Between two regressions with identical fit, $R_{a d j}^{2}$ will favour the one with the least variables therefore avoiding overfitting.

- The F-test characterizes the significance of a linear regression model compared to a derived model with at least one less coefficient. In this case, the comparison is done with a theoretical regression model without any coefficients. If the F-test p-value is lower than the acceptance rate, the $\mathrm{F}$ statistic is considered relevant and the higher the value, the higher the significance of the regression.

- The Akaike Information Criterion (AIC) assesses the goodness of fit of a model, based on the likelihood, with a penalty for the number of variables to avoid overfitting. AIC is preferred here to Bayesian Information Criterion (BIC) as Yang (2005)showed that the AIC had better results than the BIC in linear regression model selection.

\section{Sensitivity analysis}

As mentioned earlier, the building configurations on Energy Plus have been designed to follow an LHS scheme allowing both a thorough exploration of the parameter space and the calculation of sensitivity indices. To do the latter, the RBD-FAST method as described in Goffart et al. (2017) is used. The authors also underline that the RBD-FAST method is particularly suitable as it requires less samples than other variance based methods and still achieve satisfactory robustness and reliability. Finally, the fact that 2nd order sensitivity indices cannot be calculated with the RBD-FAST analysis is not a drawback in this work, as the LHS sampling of the parameter space allows a visual exploration of the 2 nd order effects, if they exist.

The sensitivity analysis inputs are the 7 properties sampled. Analysis on the coefficients themselves would barely be interpretable as the inputs vary over large intervals. This is why the sensitivity analysis focuses on the error to the theoretical HTC, $H_{d}$ or $H_{u}$, revealing which building properties have an influence on a wrong estimation of these heat transfer coefficients.

Figure 3 shows the convergence of the normalized mean and normalized variance of the error on the HTC estimation as well as the convergence of the most significant sensitivity index. Convergence is shown for Models 1 (least complex of the study) and 3 (most complex). Convergence is reached as soon as the indices are steady. In this study, it can be seen afterwards that more than 350 samples were necessary to reach convergence, which is why an initial draw of 450 samples ensured robust results. 

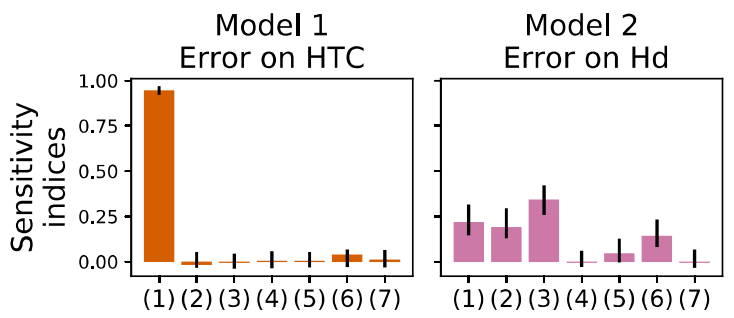

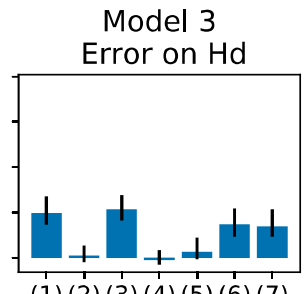

(1) (2) (3) (4) (5) (6) (7)

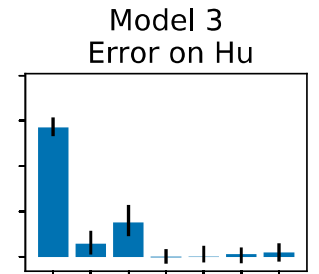

(1) (2) (3) (4) (5) (6) (7)

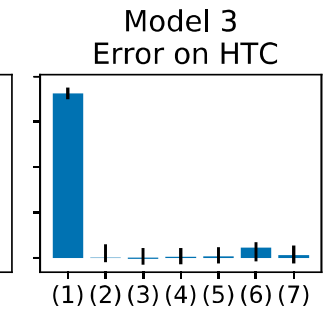

Model 3

(3) Roof Insulation Thickness

(6) Gazing Solar Transmittance
(1) Ground Floor Thermal Resistance

(4) Exterior Ground Reflectance

(2) Wall Insulation Thickness

(5) Window Height

Figure 4: Sensitivity indices of the relative error on the estimation of $H T C, H_{d}$ and $H_{u}$.
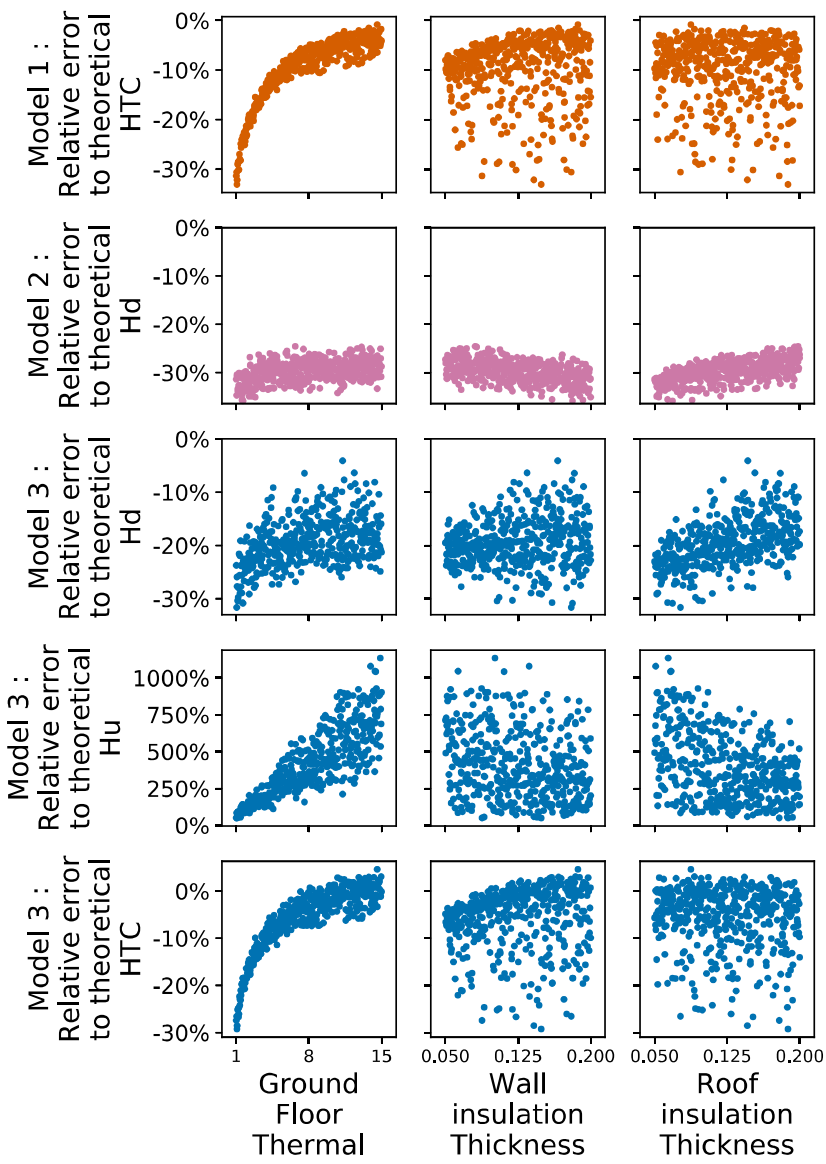
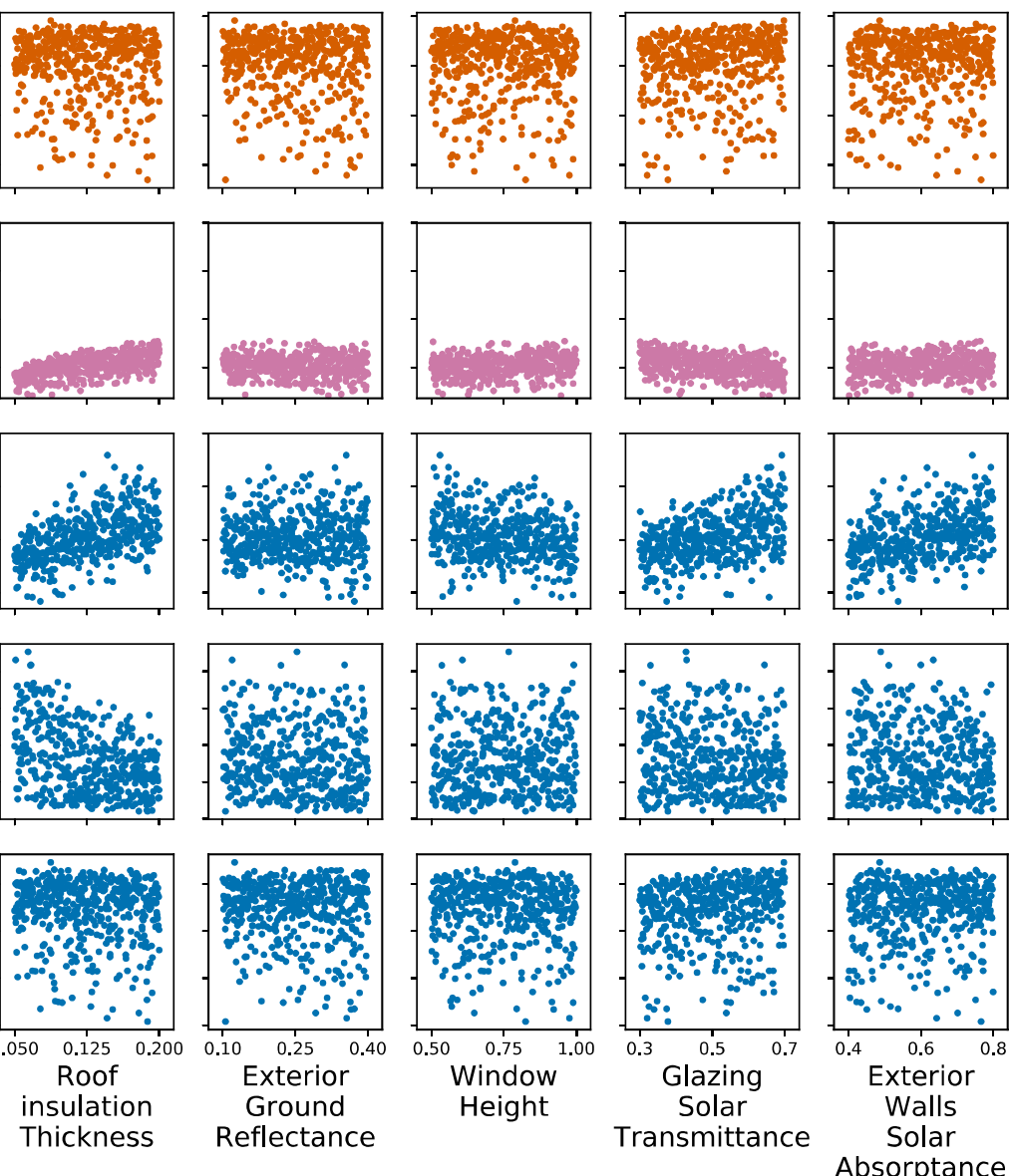

Figure 5: Variability of the $H T C, H_{d}$ and $H_{u}$ estimation regarding each property of the reference building.

Finally, to assess the robustness of the sensitivity indices, a bootstrap of 1000 draws with replacement is used. The result is shown as confidence intervals in Figure $4:$ the larger the interval, the more uncertain the value of the index. Noteworthy, as some of the intervals are almost 0.2 wide, any index lower than 0.1 is not significant and the indices should only be interpreted in comparison to one another.

\section{Results}

\section{Identifiability assessment}

The numerical procedure aims at comparing the identifiability of each model, rendered by two figures here. First the sensitivity analysis of the HTC, $H_{d}$ or $H_{u}$ estimation are shown in Figure 4. Then, the relative errors, with respect to each of the 7 properties of the reference building, are shown in Figure 5.

The sensitivity indices pictured in Figure 4 show how the variability of the error of Model 1 is extremely influenced by the variability of thermal resistance between the heated space and the basement. Indeed, according to Figure 5, the lower the insulation, the worst the error on the HTC estimation with under-estimations down to $-30 \%$. This justifies the addition of a term to the linear regression models, to explicitly represent heat loss through the basement either through a constant term (Model 2) or as a dependence to the basement temperature (Model 3).

The relative estimation error of $H_{d}$ by Models 2 and 3 is less influenced by the variability of the thermal 

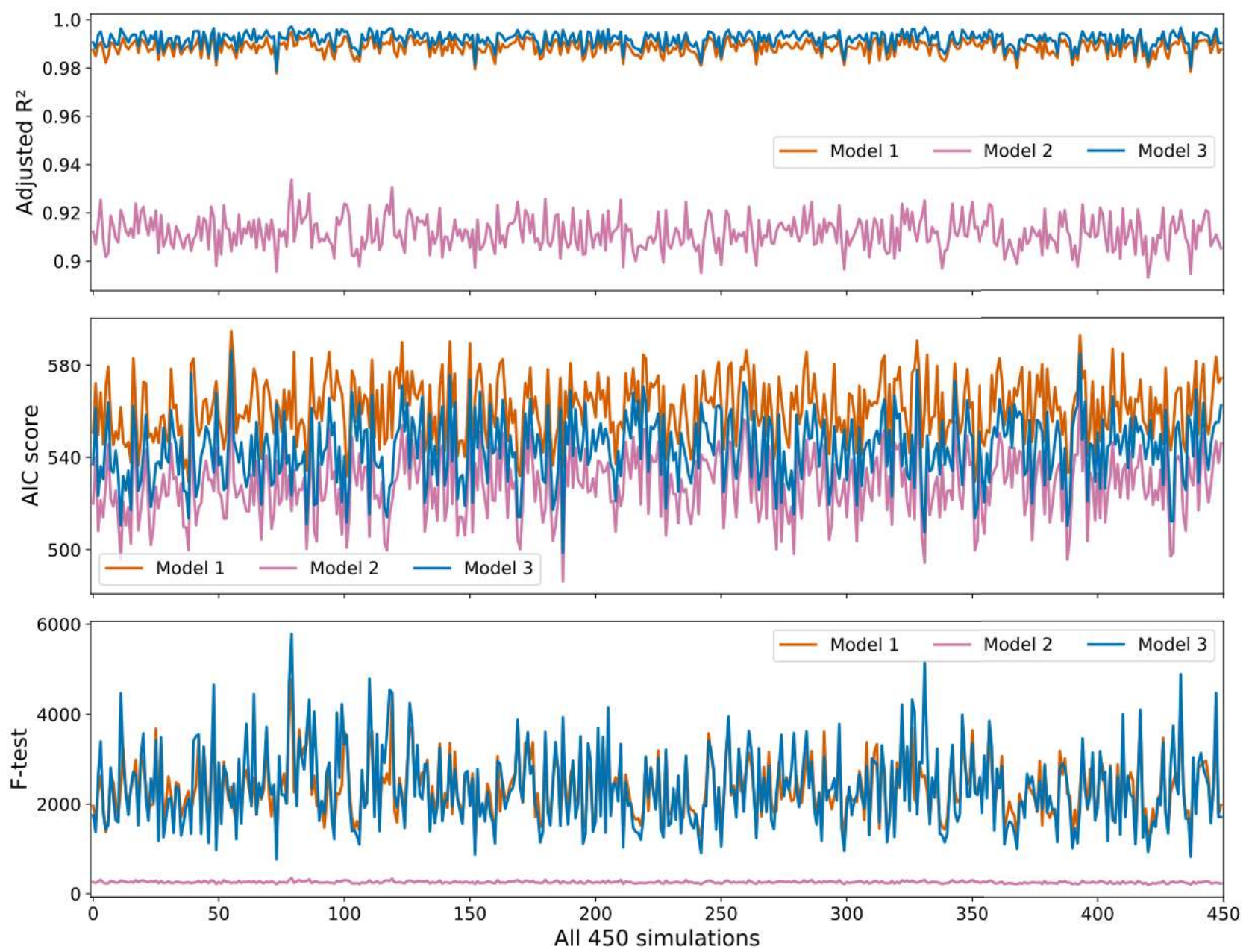

Figure 6: Comparison of all 3 models according to: adjusted $R^{2}$, AIC, F-test.

resistance of the ground floor. Both models however show a significant and systematic under-estimation of $H_{d}$. The relative error on the estimation of $H_{d}$ by Model 2 has a low variance: the error is not spread, centered around $-30 \%$ with a absolute minimum of $20 \%$ error. In none of the 450 building configurations does Model 2 recover an accurate $H_{d}$, let alone a correct HTC estimation.

Model 3, with two separate coefficients meant to represent $H_{d}$ and $H_{u}$, also shows inaccurate results regarding the estimation of disaggregated heat transfer coefficients. $H_{d}$ is systematically underestimated by 8 to $10 \%$ at best, and in the case of low ground floor thermal resistance, under-estimated by 20 to $30 \%$. On the contrary, as can be seen in Figure 5, the coefficient supposedly representing $H_{u}$ is extremely over-estimated and bears therefore no physical meaning.

The estimation of HTC with Model 3 (Equation 2) is in overall in good agreement with the theoretical value of HTC, as was Model 1, and Figure 7 indicates that Model 3 is even closer to the theoretical HTC than Model 1. In Figure 5 can be seen that similarly to Model 1, the estimation of HTC by Model 3 performs poorly when the ground floor is poorly insulated. This means that without prior knowledge of the envelope performance and in particular of the state of ground floor insulation, any HTC estimation from either Model 1 or Model 3 should be carefully interpreted. In any case, the results show that none of the 3 models enable the disaggregation of heat losses through the basement from heat losses through the rest of the envelope.

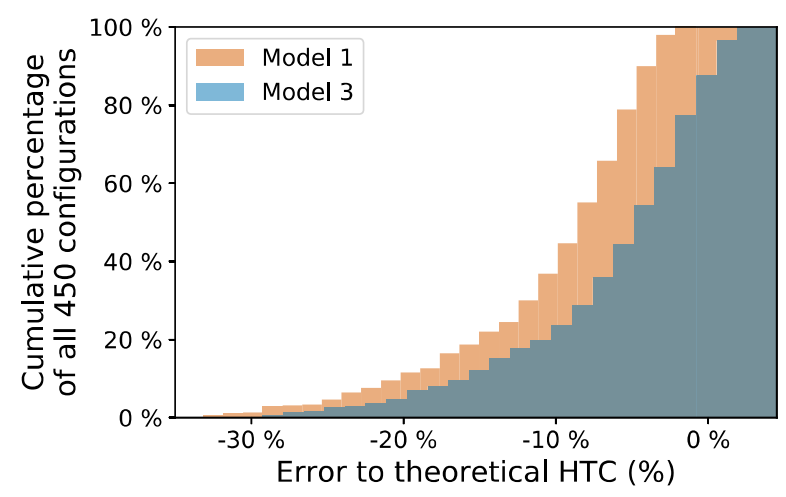

Figure 7: Cumulative histogram of the error to theoretical HTC for Models 1 and 3. 


\section{Model comparison by statistical indicators}

All 3 models are compared in Figure 6 with the $R_{a d j}^{2}$, the Fisher test and the AIC. Let us emphasize that the usual procedure for estimating parameters from data (Madsen et al., 2015) is to first select an appropriate model with these statistical tools. The underlying hypothesis is that the model fitting the best the data most probably describes more accurately the physics and has therefore most probably physically interpretable parameters.

The first observation in Figure 6 is the poor performance of Model 2. In each of the 450 configurations, it shows a significantly lower $R_{a d j}^{2}$ and a much lower F-test. Interestingly, the AIC would on the contrary favour the choice of this model among the 3 models, meaning it has a higher likelihood. Models 1 and 3 show similar performance regarding the $R_{a d j}^{2}$ and the F-test. Between both, the AIC would favour Model 3.

\section{Discussion}

The comparison of all 3 models has yielded contradictory outcomes. On the one hand, the sensitivity analysis on Model 1 justifies the addition of a term representing heat loss through the unheated basement as in Model 2 and 3 . The standard statistical indicators, all accounting for the number of variables to avoid overfitting, designate Model 3 as obtaining the best fit with the data.

On the other hand, the identifiability assessment shows how Models 2 and 3 precisely perform poorly when it comes to estimating $H_{d}$ or $H_{u}$. Between all 3 models, the identifiability assessment would make Models 1 and 3 most reliable to assess an overall heat transfer coefficient, with the exception of configurations with poor thermal resistance to the basement.

Regarding this apparent contradiction, let us remind that the model comparison tools, and particularly the AIC, have originally been designed to assess prediction performance (Yang, 2005). According to the AIC, Model 2 has the best prediction performance, followed by Models 3 and 1 . Since Models 1 and 3 are selected by the $R_{a d j}^{2}$ coefficient and the F-test, Model 3 would have been chosen in any of the 450 configurations.

It seems that there is no causality between the prediction performance of a model and the interpretability of their parameters. None of the model selection tools allow uncovering the identifiability of $H_{d}$ or $H_{u}$. This result is consistent with Chong and Menberg (2018), who showed that it is misleading to trust parameter estimation solely based on the ability of a model to predict test data. Similarly, prediction is not guaranteed to be most satisfactory with the most identifiable models.

Despite the use of a 5 weeks dataset, it cannot come as a surprise that Models 1 and 3 only yield satisfactory estimates for an overall HTC, whereas literature review (Bauwens and Roels, 2014) suggested a priori more accurate results with among others regression models analogous to Model 2. It is indeed our belief that coheating tests provide data better fitted for regression models, even with smaller datasets. Non intrusive sensors and non intrusive measurement plan deliver indeed less informative data than a coheating test and make it more dependent on weather conditions (Deconinck and Roels, 2017). Therefore, it would be pertinent to improve the way solar gains are represented in the regression models.

Finally, measuring the air temperature in the basement and including it as an explanatory variable in the regression does not allow disaggregating between direct loss to the outdoor and loss through the basement. If however the objective is to make this distinction, the results suggest that such a light measurement plan is insufficient. More intrusive measurements and/or more complex models might enhance the identifiability of $H_{d}$ and $H_{u}$, such as heat flow measurement through the part of the envelope, as done in Bauwens and Roels (2017) in the case of adjacent spaces. Finally, the results in Deconinck and Roels (2017) suggest that more complex models such as dynamic state space models could perform better while still exploit data from a non intrusive measurement plan.

\section{Conclusion}

The paper describes a new and thorough way to study the identifiability of any model, based on a numerical procedure allowing to test many building configurations. The paper details how the procedure is carried out on regression models and how accurately these models can estimate heat transfer coefficients in the particular case of buildings with unheated basements.

The results show that the two out of the three models tested achieve satisfactory estimation of the overall HTC when the ground floor of the building is well insulated, but that none of the regression models studied is suited when poorly insulated. The regression models tested are even less able to distinguish direct losses to the ambient environment from losses to the basement. Future work will focus on assessing how dynamical models perform with similar non intrusive measurement plans.

\section{Acknowledgements}

The authors would like to thank the French National Research Agency (ANR) for funding this work through the BAYREB research project (ANR-15CE22-0003). 


\section{References}

Atlanta: ASHRAE (2001). International Weather for Energy Calculations (IWEC Weather Files) Users Manual and CD-ROM.

Bacher, P. and H. Madsen (2011). Identifying suitable models for the heat dynamics of buildings. Energy and Buildings 43(7), 1511-1522.

Bauwens, G. and S. Roels (2014). Co-heating test: A state-of-the-art. Energy and Buildings 82, 163-172.

Bauwens, G. and S. Roels (2017). On site thermal performance characterization of building envelopes: How important are heat exchanges with neighbouring zones. Energy Procedia 132, 339-344.

Chambers, J. D. and T. Oreszczyn (2019). Deconstruct: A scalable method of as-built heat power loss coefficient inference for uk dwellings using smart meter data. Energy and Buildings 183, 443-453.

Chong, A. and K. Menberg (2018). Guidelines for the bayesian calibration of building energy models. Energy and Buildings 174, 527-547.

Deconinck, A.-H. and S. Roels (2017). Is stochastic grey-box modelling suited for physical properties estimation of building components from on-site measurements? Journal of Building Physics 40(5), $444-471$.

Everett, R. (1985). Rapid thermal calibration of houses. Milton Keynes, UK: Open University Energy Research Group ERG 55.

Farmer, D., D. Johnston, and D. Miles-Shenton (2016). Obtaining the heat loss coefficient of a dwelling using its heating system (integrated coheating). Energy and Buildings 117, 1-10.

Goffart, J., M. Rabouille, and N. Mendes (2017). Uncertainty and sensitivity analysis applied to hygrothermal simulation of a brick building in a hot and humid climate. Journal of Building Performance Simulation 10(1), 37-57.

International Organisation for Standardisation (2017a). Thermal performance of buildings - Heat transfer via the ground - Calculation methods (ISO 13370).

International Organisation for Standardisation (2017b). Thermal performance of buildings - Transmission and ventilation heat transfer coefficients - Calculation method (ISO 13789).

Jack, R., D. Loveday, D. Allinson, and K. Lomas (2018). First evidence for the reliability of building co-heating tests. Building Research \& Information 46(4), 383-401.
(2016). Reliable building energy performance characterisation based on full scale dynamic measurements : $S T$ b Overview of methods to analyse dynamic data.

National Renewable Energy Lab., Golden, CO (US) (1995). International Energy Agency building energy simulation test (BESTEST) and diagnostic method.

Madsen, H. (2007). Time series analysis. Chapman and Hall/CRC.

Madsen, H., P. Bacher, G. Bauwens, A.-H. Deconinck, G. Reynders, S. Roels, E. Himpe, and G. Lethé (2015). Thermal Performance Characterization using Time Series Data -IEA EBC Annex 58 Guidelines Thermal performance characterization using time series data -statistical guidelines IEA EBC Annex 58.

Maiwald, T., J. Timmer, C. Kreutz, U. Klingmüller, and A. Raue (2011). Addressing parameter identifiability by model-based experimentation. IET Systems Biology 5(2), 120-130.

Senave, M., G. Reynders, S. Verbeke, and D. Saelens (2017). A simulation exercise to improve building energy performance characterization via on-board monitoring. Energy Procedia 132, 969-974.

Stamp, S., R. Lowe, and H. Altamirano-Medina (2013). An investigation into the role of thermal mass on the accuracy of co-heating tests through simulations \& field results. In Proceedings of BS2013: 13th Conference of international Building Performance Simulation Association (IPBSA), Building Simulation, pp. 25-28.

Yang, Y. (2005). Can the strengths of aic and bic be shared? a conflict between model indentification and regression estimation. Biometrika 92(4), 937950 . 